\title{
Management of Postoperative Neurosurgical Patients
}

\author{
Rajeev Chauhan ${ }^{1}$ Summit Dev Bloria ${ }^{1}$ Ankur Luthra ${ }^{1}$ \\ ${ }^{1}$ Department of Anaesthesia and Intensive Care, Postgraduate \\ Institute of Medical Education and Research (PGIMER), Chandigarh, \\ Punjab, India \\ Address for correspondence Ankur Luthra, MD, DM Neuroanaesthesia, \\ H. No. 979, Aashirwad Enclave, Sector 49-A, Chandigarh, 160047, \\ Punjab, India (e-mail: zazzydude979@gmail.com).
}

Indian J Neurosurg 2019;8:179-184

\begin{abstract}
Keywords

- neurosurgical

- postoperative

- management

The patient profile undergoing neurosurgery varies from neonates to elderly, and from patients undergoing elective surgery to patients undergoing emergency surgeries. The goals of postoperative management include prevention of secondary brain injury and taking care of the major organ systems till the time patient recovers from the primary insult. Postsurgery, patients may be shifted to a neurosurgical intensive care unit or managed in a neurosurgical ward. As a general rule, all patients should be nursed 30-degree head up. We will be discussing the basic principles of postoperative management of neurosurgical patients.
\end{abstract}

\section{Introduction}

The domains of neurosurgery have extended over time so that now any discussion about the postoperative care of neurosurgical patients must include the following categories of patients:

1. Those who have undergone intracranial surgery under general anesthesia (GA), under monitored anesthesia care (MAC), and under sedation.

2. Those who have undergone spinal procedures.

3. Those who have undergone neuroradiology procedures under GA, MAC, or sedation.

4. Those who have undergone gamma knife surgery.

Postoperatively, these patients can be cared for either in an intensive care unit (ICU) or in high-dependency wards.

\section{Deciding the Level of Care and Monitoring Postoperatively}

The postoperative level of care and monitoring should ideally be decided preoperatively. The decision whether to shift the patient to an ICU on mechanical ventilation or whether to extubate inside operation theater depends on several factors which include the following:

1. Preoperative status of patient.

2. Site of the surgery.

3. Surgical factors such as bleeding, and any brainstem handling.

4. Specific anticipated postoperative needs of the patient.
The following risk factors for postoperative shifting to ICU have been suggested:

1. Diabetes mellitus.

2. Duration of surgery more than 4 hours.

3. Surgery in lateral decubitus position.

4. Older age.

Rhondali et al conducted an observational cohort study involving 358 patients admitted to neuro-ICU after elective intracranial procedures. They observed that the predictors of postoperative complications included failure to extubate the trachea in operating room, and, to a lesser extent, a duration of surgery of more than 4 hours and lateral positioning of the patient during the procedure. ${ }^{1}$ Another prospective cohort study involving 400 patients suggested that diabetes and older age predicted the need for ICU admission postoperatively. ${ }^{2}$

Some common problems and conditions encountered in the postoperative period in neurosurgical patients are as follows:

1. Delayed awakening post surgery: It can have surgical or anesthetic causes. Generally, the effect of most anesthetic agents gets dissipated by 30 to 60 minutes. Any delayed awakening beyond this time period in usual circumstances should suggest a surgical cause.

2. Seizures: Early postoperative seizures are caused due to the surgical injury and stress response, whereas late postoperative seizures represent epilepsy and require long-term seizure prophylaxis.

(C2019 Neurological Surgeons' Society of India
License terms

$($ () (1) $\odot \circledast$
May 4, 2019

accepted after revision

July 8, 2019

published online

September 24, 2019
DOI https://doi.org/

$10.1055 / \mathrm{s}-0039-1698001$

ISSN 2277-954X. 
3. Electrolyte abnormalities: All electrolyte abnormalities can be seen postoperatively, the most common being hyponatremia and hypernatremia. ${ }^{3}$ The two major causes of hyponatremia include syndrome of inappropriate antidiuretic hormone secretion (SIADH) and cerebral salt wasting syndrome (CSWS), which must be differentiated as the management of these conditions is entirely different. Dyselectrolytemias can sometimes occur after surgeries for pituitary tumors. ${ }^{4}$

4. Tension pneumocephalus: It is a life-threatening condition, identified by "Mount Fuji" sign or "air bubble sign" on noncontrast computed tomography (CT) scan of the head. The patient may present with deterioration of consciousness, focal neurological deficits, severe restlessness, or seizures. Tension pneumocephalus must be rapidly treated with $100 \%$ oxygen and a frontal burr hole to release the trapped air under local or general anesthesia.

5. Postoperative infection: Risk factors for postoperative infection after cranial surgery include altered sensorium, multiple operations, pre-existing infection, emergency surgery, duration of surgery more than 4 hours, urinary catheterization, cerebrospinal fluid (CSF) leak, and prolonged ventilatory support. ${ }^{5}$

Postoperative meningitis (POM) remains one of the most dreaded complications post neurosurgery. The reported incidence, according to most literature, of postoperative meningitis is quite variable between 0.5 and $8 \%{ }^{6.7} \mathrm{~A}$ high index of suspicion for meningitis is essential in patients who have the clinical triad of fever, neck stiffness, and disturbance in sensorium during the postoperative period. Postoperative bacterial meningitis is a rare complication of spinal surgery. It is considered to be a complication related to intraoperative incidental durotomy.

The etiology of POM includes a wide spectrum of microorganisms from gram-positive cocci to gram-negative bacilli. Postoperative aseptic meningitis has also been described. Aseptic meningitis occurs more frequently in children and after surgery involving the posterior fossa, and it may occur as a result of a local inflammatory reaction to blood breakdown products or to tumor antigens. ${ }^{8,9}$

Chen et al in a retrospective cohort study described diabetes and presence of CSF shunts as high-risk factors for development of postoperative meningitis. They found Acinetobacter baumannii, Enterococcus sp, Streptococcus intermedius, and Klebsiella pneumoniae as the most common organisms responsible for postoperative meningitis. ${ }^{10}$

According to the study by Srinivas et al, involving more than 18,000 patients, the incidence of postoperative meningitis was $2.2 \%$. Gram-negative organisms were the most common causative pathogens and postoperative meningitis carried an overall mortality of $5 \%{ }^{11}$

Another retrospective study involving more than 1,000 patients of endonasal endoscopic skull base surgery found that the incidence of postoperative infection in endoscopic surgeries was comparable with that in open craniotomies. ${ }^{12}$

6. Intracranial hemorrhage (ICH): An important and dangerous postoperative event is ICH. Most of the cases of ICH occur within 6 hours of surgery and are associated with postoperative hypertension and coagulopathy. Seifman et al in a review suggested that the incidence of postoperative hematoma varied between 0.8 and $6.9 \% .13$

7. Ventilator associated pneumonia (VAP): In patients who require prolonged mechanical ventilation (cervical spine, poor Glasgow Coma Scale (GCS) preoperatively) or those who suffer from aspiration owing to lower cranial nerve palsy with absent gag and cough reflex, even though tracheostomized early, ventilator-associated pneumonia develops as a major management issue. VAP is defined as pneumonia in any patient who is intubated and ventilated at the time of or within 48 hours before the onset of the event. It has been documented that simple measures such as the use of an alcohol-based hand rub before endotracheal suctioning reduces the incidence of VAP. ${ }^{14}$

VAP is classified as early (occurring within first 4 days of hospitalization) or late (occurring on or after 5 days of hospitalization).

Clinical pulmonary infection score (CPIS) is a tool that was described to objectify the diagnosis of patients with suspected VAP. CPIS combines clinical, radiographic, physiological $\left(\mathrm{PaO}_{2} / \mathrm{FiO}_{2}\right)$, and microbiologic data into a single numerical result. CPIS $>6$ has demonstrated a good correlation with the presence of pneumonia as defined by quantitative cultures of bronchoscopic and nonbronchoscopic broncho-alveolar lavage (BAL) specimens. ${ }^{15}$

The two most commonly used methods for the microbiological confirmation of VAP are bronchoalveolar lavage with quantitative cultures and endotracheal tube aspirate.

The treatment of VAP consists of delivery of early and appropriate antibiotics. Broad-spectrum antibiotic coverage should be initiated as soon as VAP is suspected and should be replaced with targeted antibiotics once pathogen susceptibility has been determined. ${ }^{16}$

\section{Monitoring in Postoperative Period}

1. Clinical monitoring-The minimum clinical monitoring in the postoperative period must consist of repeated GCS evaluation along with pupillary examination and motor and sensory examination to detect any new onset neurological deficit or deterioration. In patients who have undergone posterior fossa surgeries in close proximity to the brainstem, cranial nerve examination must be done.

2. Respiratory monitoring-Oxygen saturation must be monitored in all patients. End-tidal carbon dioxide $\left(\mathrm{EtCO}_{2}\right)$ should be measured in all intubated patients. Partial pressures of $\mathrm{CO}_{2}\left(\mathrm{PaCO}_{2}\right)$ levels should be measured upon shifting to ICU and then at regular intervals or during any neurological deterioration as both hypo- and hypercapnia may be detrimental to the already injured brain. Normal $\mathrm{PaCO}_{2}$ values to be targeted in these patients vary between 35 and $45 \mathrm{~mm} \mathrm{Hg}$.

3. Hemodynamic monitoring-Noninvasive blood pressure (BP) monitoring should be done in all patients at regular intervals. In patients with uncontrolled hypotension 
requiring vasopressor or inotropic support or patients with malignant hypertension, or in patients requiring repeated blood gas measurements, invasive beat to beat monitoring of blood pressure is mandatory.

Transthoracic echocardiography (TTE) must be readily available in situations such as unexplained hemodynamic instability, determination of volume status of the patient, and assessment of cardiac status in patients with subarachnoid hemorrhage (SAH), and traumatic brain injury (TBI).

4. Neurological monitoring-Optic nerve sheath diameter monitoring can be a useful technique to detect raised intracranial pressure (ICP) noninvasively. In patients with external ventricular drains (EVDs) in situ, direct measurement of ICP can be done.17 Transcranial Doppler (TCD) can help determine cerebral blood flow indirectly and detect vasospasm in patients with SAH. TCD has also been used to determine status of cerebral autoregulation using transient hyperemic response test (THRT). Near infrared spectroscopy (NIRS) is a device which provides information about regional tissue oxygen saturation by measurement of absorption of infrared light. It is noninvasive and can also be used to estimate cerebral blood flow using the indocyanine green dye.

5. Others-Regular monitoring of blood glucose levels must be done. Both hypo- and hyperglycemia have been associated with poor outcomes in neurological patients. Tight control of glucose $(70-110 \mathrm{mg} / \mathrm{dL})$ has not been recommended in critically ill patients as the number of missed hypoglycemic episode increases, which may lead to poor outcomes in such patients. ${ }^{18}$ Similarly, hyperglycemia episodes (>200 mg/dL) have also been proved to be detrimental and therefore adequate glucose levels of $120-180 \mathrm{mg} / \mathrm{dL}$ need to be targeted in such patients. ${ }^{19-21}$

Also, serum sodium levels and urine output must be serially measured, especially in patients who have undergone pituitary surgery or craniopharyngioma to diagnose diabetes insipidus. Perioperative short duration steroids may need to be administered to patients who had documented steroid deficiency preoperatively.

\section{Management of Common Postoperative Complications}

\section{Postoperative Nausea and Vomiting Prophylaxis}

The incidence of postoperative nausea and vomiting (PONV) after craniotomy is said to be around $50 \%{ }^{22}$ Risk factors for PONV after intracranial surgery include female gender, younger age, and infratentorial surgery. PONV can cause arterial hypertension and a consequent increase in ICP. Vomiting in a patient who is not fully conscious can prove to be catastrophic due to aspiration followed by respiratory deterioration. Most commonly used drugs for PONV prophylaxis include ondansetron, dexamethasone, and cyclizine. A commonly followed approach is to provide intraoperative PONV prophylaxis and then provide rescue therapy in the postoperative period.

\section{Postoperative Analgesia}

Provision of adequate analgesia in neurosurgical procedures remains a neglected entity. Inadequate analgesia post surgery can lead to hypertension and tachycardia. It is said that approximately $60 \%$ of postcraniotomy patients experience moderate to severe pain up to the second postoperative day or present with persistent pain with neuropathic elements several months after surgery. ${ }^{23}$ Multimodality analgesia utilizing nerve blocks, non-steroidal anti-inflammatory drugs (NSAIDS), opioids, and other drugs is considered to be the best modality for postoperative analgesia in neurosurgical patients. Intravenous opioid patient-controlled analgesia provides betterpostoperative analgesia than intermittent opioid administration. ${ }^{24}$

The use of scalp block has demonstrated lower postoperative pain scores and lower postoperative rescue drug requirement. ${ }^{25}$ The exact value of preincision wound infiltration in providing postoperative analgesia is doubtful, with some studies showing it to be effective whereas others not showing any analgesic benefit postoperatively. ${ }^{26,27}$ NSAIDS can cause platelet dysfunction and increased bleeding times while paracetamol alone is ineffective to provide adequate analgesia. Overall, although the use of NSAIDS has been shown by some authors to provide satisfactory pain relief, their use remains contentious and presently there is no consensus on their role..$^{28,29}$

\section{Thromboprophylaxis}

Mechanical thromboprophylaxis in the form of intermittent mechanical pneumatic devices should be initiated immediately post surgery. Pharmacological prophylaxis in the form of low molecular weight heparin may be started as early as 24 to 48 hours after surgery in discussion with the neurosurgeon, when a CT scan has excluded intracranial hemorrhage and the hemostasis achieved intraoperatively seems to be stable.

\section{Seizure Prophylaxis}

Patients getting antiseizure medication preoperatively should continue receiving the same postoperatively also. Routine antiseizure prophylaxis is not recommended in patients with brain tumors. The two most commonly used drugs are phenytoin and levetiracetam Considering the many side effects of phenytoin (hirsutism, osteomalacia, teratogenicity, megaloblastic anemia, ataxia, lymphadenopathy, and hyperglycemia), levetiracetam can be a better drug with lesser side effects to administer for seizure prophylaxis; however, local institutional protocols do come into play when antiseizure medications are concerned.

\section{Corticosteroids}

Dexamethasone is the most common corticosteroid administered, for management of vasogenic edema. It is usually initiated preoperatively, and continued peri- and postoperatively. A plateau effect is observed after 4 to 6 days of treatment. Whenever, such drugs are administered, regular blood sugar monitoring becomes very essential and needs to be appropriately managed as per clinical situations. 


\section{Special Considerations as per the Procedures Performed}

1. Cerebral aneurysm clipping/coiling-Good grade cerebral aneurysms who have undergone an uneventful coiling/ clipping can be safely extubated at the end of procedure. Early assessment of neurological function is imperative.

Of great importance in these patients is the early detection and management of delayed cerebral ischemia. Repeated transcranial Doppler examination help in early detection of vasospasm. ${ }^{30}$ All these patients must be administered nimodipine $60 \mathrm{mg}(1 \mathrm{mg} / \mathrm{kg})$ every 4 hours orally up to 21 days from the day of ictus.

The importance of triple $\mathrm{H}$ therapy for management of vasospasm has waned over time to an extent that presently only induced hypertension is employed as a modality for management of vasospasm. The most common drugs used for induced hypertension include noradrenaline, phenylephrine, dobutamine, and dopamine. The BP is titrated to a level necessary to reverse the features of vasospasm or to a maximum of 160 to $200 \mathrm{~mm} \mathrm{Hg}$ post securing the aneurysm. Many patients with vasospasm may require administration of targeted intra-arterial nimodipine therapy after the confirmation by digital subtraction angiography.

In poor grade cerebral aneurysms, fluid and electrolyte abnormalities are exceedingly common and must be detected and corrected early. Transthoracic echocardiography can be used to detect cardiac manifestations of aneurysmal SAH.

In patients who have undergone aneurysm coiling, the arterial puncture site and the distal pulses must be examined at regular intervals.

2. Surgeries for pituitary tumors-The surgeries for pituitary tumors can be performed transcranially or transnasally. The postoperative pain scores and analgesic requirements are considered to be lower in patients who have undergone trans-sphenoidal surgery. The acromegaly patients need to be monitored regularly to detect any airway obstruction or impending respiratory distress. All patients receiving hormone supplementation preoperatively must be restarted on the drugs postoperatively as soon as possible. CSF rhinorrhea is a possibility in these patients if arachnoid has been breached and a thorough monitoring of CSF leak, rising total leucocyte count and fever must be investigated further to prevent and treat occurrence of meningitis. Apart from CSF leak, diabetes insipidus, postoperative nausea and vomiting, and hematoma at the operation site are other commonly found postoperative complications. ${ }^{31}$

3. Stroke patients-While caring for stroke patients who have undergone mechanical thrombectomy, it is important to realize that good postoperative care is necessary to optimize outcomes. These patients are critically ill and need to be cared for postoperatively, especially with regard to issues such as stress ulcers, pressure ulcers, and peripheral venous thrombosis. Also, hyperglycemia, hyperthermia, and pulmonary aspiration should be prevented. Postoperative BP goals should balance penumbra perfusion against risk for reperfusion injury and hemorrhage. A mean arterial pressure of 10 to $20 \%$ above baseline (systolic blood pressure (SBP) $<185 \mathrm{~mm} \mathrm{Hg}$ ) to support ischemic penumbra, but a lower SBP $(140-160 \mathrm{~mm} \mathrm{Hg})$ is necessary following intracranial hemorrhage. Other considerations include management of possible allergic reactions to contrast agents and prevention of contrast-induced nephropathy. The site of arterial puncture must be monitored regularly to detect any bleeding, or formation of pseudoaneurysm early. Close, frequent neurological monitoring, especially in the first 24 to 48 hours is essential as these patients can suffer from vessel reocclusion postoperatively.

4. Traumatic brain injury-The postoperative care of patients who have undergone decompression craniectomy becomes a part of the critical care of these patients. The primary aim in these patients who have suffered traumatic brain injury is to prevent secondary brain insults and to maintain cerebral homeostasis. Maintenance of a CPP of at least $70 \mathrm{~mm} \mathrm{Hg}$ should be carefully sought for to prevent secondary brain ischemia (Brain Trauma Foundation guidelines, fourth edition). The use of hyperventilation in these patients should be limited to periods of impending neurological deterioration as prolonged hyperventilation may devoid the penumbral regions of essential blood and oxygen supply due to vasoconstriction. Hyperventilation should be avoided in first 24 hours post injury as the brain is already having a critically low blood supply during this period. It is imperative that some kind of ICP monitoring (invasive or noninvasive) must be continued postoperatively in these patients.

Nutrition should be started in these patients as soon as possible. By fifth day, patients should attain basal caloric replacement. While sedating these patients, it must be remembered that high doses of propofol can produce significant morbidity (propofol infusion syndrome).

Parkland protocol: This protocol helps us guide timing of DVT prophylaxis in patients of TBI. In this algorithm, patients with any of the following are classified "low risk" for spontaneous progression: subdural hemorrhage $<8 \mathrm{~mm}$ thick, epidural hemorrhage $<8 \mathrm{~mm}$ thick, contusions $<20 \mathrm{~mm}$ in diameter, a single contusion per lobe, any amount of subarachnoid hemorrhage, or any amount of intraventricular hemorrhage. Patients with any injury exceeding these are "high risk" for progression. In patients at low risk, enoxaparin can be initiated 24 hours post injury, provided CT head performed at 24 hours post injury shows a stable injury. In patients at high risk, a CT head should be performed after 72 hours of injury and enoxaparin should be initiated only after CT head reveals a stable injury.

Around 5 to $7 \%$ percent of patients hospitalized for TBI experience seizures..$^{32}$ The latest guidelines issued by the Brain Trauma Foundation and the American Academy of Neurology (AAN) recommend post traumatic seizures (PTS) prophylaxis only during the first 7 days after TBI.

TBI can result in coagulopathy, stress ulcers, pituitary dysfunction, neurogenic pulmonary edema, pulmonary 
aspiration, and other systemic manifestations which must be kept in mind when caring for these patients.

6. Spine surgery patients-Most patients can be safely extubated post spinal surgery. In patients who have ndergone a cervical surgery, a long duration surgery in prone position, having preoperative pulmonary impairment or patients with metabolic impairment, extubation may be delayed. The nerve supply of diaphragm comes from phrenic nerve (C3-C5). Respiratory and pulmonary function may be significantly affected following cervical or thoracic spinal cord injury. Injury to spinal cord at or above the level of C3 causes apnoea and complete loss of respiratory function, and these patients require long-term mechanical ventilation. In cervical spinal cord injury below $\mathrm{C}$, there will be partial diaphragmatic paralysis and the ability of the patient to cough is highly impaired. Hence, these patients rapidly develop hypercarbia, hypoxemia, atelectasis, and inability to clear their secretions. Therefore, nearly all patients with a complete subaxial cervical spinal cord injury (SCI) will require tracheostomy, and mechanical ventilatory support during the early phases of therapy. Nearly all patients with complete cervical SCI above $\mathrm{C} 6$ require a tracheostomy and a tracheostomy tube should be placed early (3-7 days) from the time of injury, as little benefit is realized by delaying the procedure further ${ }^{33}$ In patients on mechanical ventilation, all precautions must be taken to prevent development of ventilator-induced lung injury and ventilator-associated pneumonia with the help of lung protective ventilation, chest physiotherapy, and maintenance of good oral hygiene. It must be remembered that pulmonary complications are the most common cause of early mortality and morbidity in these patients. In most patients with lesions below $\mathrm{C} 4$, weaning from ventilator should be possible eventually after around 2 to 3 weeks of ventilation.

Autonomic dysreflexia (AD) is a real threat in postoperative period and can be life threatening. The most common symptoms of autonomic dysreflexia are sweating, pounding headache, tingling sensation on the face and neck, blotchy skin around the neck, and goose bumps. A variety of nonnoxious or noxious stimuli can trigger episodes of $A D$, most common being irritation of the urinary bladder or colon. Briefly, management consists of raising the head end of the bed, loosening of clothes, and removing compression stockings and abdominal binder. The trigger should be detected and removed. Pharmacological control of BP should be done.

Postoperative visual loss is a rare but tragic complication which is seen in around $0.2 \%$ of spine patients. ${ }^{34}$ Hence, vision should be assessed after prolonged surgery after the patient becomes awake and alert. Any indication of deficit should be immediately followed by an ophthalmologic consultation. Magnetic resonance imaging should also be considered to evaluate for nonophthalmologic and intracranial causes of blindness.

Venous thromboembolism should be prevented and intermittent pneumatic compression with pharmacologic prophylaxis should be administered once adequate hemostasis is obtained (preferably within 48-72 hours following surgery). ${ }^{35}$
Surgical site infections can occur post spine surgery. The risk factors include diabetes, history of smoking, and alcohol intake.

7. Others-Patients who have undergone procedures under MAC or sedation (stereotactic biopsy, awake craniotomy, etc.) must be monitored for development of any respiratory embarrassment.

In patients who have undergone endoscopic third ventriculostomy, transient neurological deficit is common. ${ }^{36}$ Postoperative monitoring of serum electrolyte levels is warranted because diabetes insipidus and hypothalamic dysfunction have been reported in multiple series of patients undergoing endoscopic surgery. ${ }^{37}$

Postoperative behavioral and cognitive problems are common after DBS insertion and should be managed with assessment of potential underlying causes. ${ }^{38}$

Normal perfusion pressure breakthrough is a common occurrence in patients who have been treated for arteriovenous malformations or carotid endartectomy, leading to significant brain swelling. These patients can suffer from hemodynamic changes postoperatively and intra-arterial cannula is maintained during the initial postoperative period to permit continuous blood pressure monitoring and blood sampling for arterial blood gas analyses. TCD monitoring into the postoperative period has been used to detect microemboli originating in the ipsilateral carotid artery in the hours following CEA.

\section{Conclusion}

Postoperative care of neurosurgical patients remains a risky affair, which must be managed with utmost care. While a proper postoperative care in neurosurgical patients will ensure speedy recovery and better outcomes, any mishap in the postoperative period will undo all the efforts made on the patient by the intraoperative team.

\section{Conflict of Interest}

None declared.

\section{References}

1 Rhondali O, Genty C, Halle C, et al. Do patients still require admission to an intensive care unit after elective craniotomy for brain surgery? J Neurosurg Anesthesiol 2011;23(2):118-123

2 Hanak BW, Walcott BP, Nahed BV, et al. Postoperative intensive care unit requirements after elective craniotomy. World Neurosurg 2014;81(1):165-172

3 Tisdall M, Crocker M, Watkiss J, Smith M. Disturbances of sodium in critically ill neurologic patients: a clinical review. J Neurosurg Anesthesiol 2006;18:57-63

4 Kristof RA, Rother M, Neuloh G, Klingmüller D. Incidence, clinical manifestations, and course of water and electrolyte metabolism disturbances following transsphenoidal pituitary adenoma surgery: a prospective observational study. J Neurosurg 2009;111(3):555-562

5 Patir R, Mahapatra AK, Banerji AK. Risk factors in postoperative neurosurgical infection. A prospective study. Acta Neurochir (Wien) 1992 
6 Tenney JH, Vlahov D, Salcman M, Ducker TB. Wide variation in risk of wound infection following clean neurosurgery. Implications for perioperative antibiotic prophylaxis. J Neurosurg 1985;62(2):243-247

7 Sharma MS, Vohra A, Thomas P, et al. Effect of risk-stratified, protocol-based perioperative chemoprophylaxis on nosocomial infection rates in a series of 31927 consecutive neurosurgical procedures (1994-2006) Neurosurgery 2009;64(6):1123-1130, discussion 1130-1131

8 Carmel PW, Greif LK. The aseptic meningitis syndrome: a complication of posterior fossa surgery. Pediatr Neurosurg 1993;19(5):276-280

9 Finlayson AI, Penfield W. Acute postoperative aseptic leptomeningitis. Arch Neurol Psychiatr 1941;46:250-76

10 Chen C, Zhang B, Yu S, et al. The incidence and risk factors of meningitis after major craniotomy in China: a retrospective cohort study. PLoS One 2014;9(7):e101961

11 Srinivas D, Veena Kumari HB, Somanna S, Bhagavatula I, Anandappa $\mathrm{CB}$. The incidence of postoperative meningitis in neurosurgery: an institutional experience. Neurol India 2011;59(2):195-198

12 Kono Y, Prevedello DM, Snyderman CH, Gardner PA, Kassam $A B$, Carrau RL, et al. One thousand endoscopic skull base surgical procedures demystifying the infection potential: incidence and description of postoperative meningitis and brain abscesses. Infect Control Hosp Epidemiol 2011;32(1):77-83

13 Seifman MA, Lewis PM, Rosenfeld JV, Hwang PY. Postoperative intracranial haemorrhage: a review. Neurosurg Rev 2011;34(4):393-407

14 Saramma PP, Krishnakumar K, Dash PK, Sarma PS. Alcohol-based hand rub and ventilator-associated pneumonia after elective neurosurgery: an interventional study. Indian J Crit Care Med 2011;15(4):203-208

15 Pugin J, Auckenthaler R, Mili N, Janssens JP, Lew PD, Suter PM. Diagnosis of ventilator-associated pneumonia by bacteriologic analysis of bronchoscopic and nonbronchoscopic "blind" bronchoalveolar lavage fluid. Am Rev Respir Dis 1991;143(5 Pt 1):1121-1129

16 Joffe AR, Muscedere J, Marshall JC, Su Y, Heyland DK; Canadian Critical Care Trials Group. The safety of targeted antibiotic therapy for ventilator-associated pneumonia: a multicenter observational study. J Crit Care 2008;23(1):82-90

17 Ehtisham A, Taylor S, Bayless L, Klein MW, Janzen JM. Placement of external ventricular drains and intracranial pressure monitors by neurointensivists. Neurocrit Care 2009;10(2):241-247

18 Finfer S, Chittock DR, Su SY, et al. NICE-SUGAR Study Investigators. Intensive versus conventional glucose control in critically ill patients. N Engl J Med 2009;360(13):1283-1297

19 Lam AM, Winn HR, Cullen BF, Sundling N. Hyperglycemia and neurological outcome in patients with head injury. J Neurosurg 1991;75(4):545-551

20 Bruno A, Biller J, Adams HP Jr, et al. Acute blood glucose level and outcome from ischemic stroke. Trial of ORG 10172 in acute stroke treatment (TOAST) investigators. Neurology 1999;52(2):280-284

21 Kimura $\mathrm{K}$, Iguchi $\mathrm{Y}$, Inoue $\mathrm{T}$, et al. Hyperglycemia independently increases the risk of early death in acute spontaneous intracerebral hemorrhage. J Neurol Sci 2007;255(1-2):90-94
22 Audibert G, Vial V. Postoperative nausea and vomiting after neurosurgery (infratentorial and supratentorial surgery) [in French]. Ann Fr Anesth Reanim 2004;23(4):422-427

23 Leslie K, Troedel S, Irwin K, et al. Quality of recovery from anesthesia in neurosurgical patients. Anesthesiology 2003;99(5):1158-1165

24 Bollish SJ, Collins CL, Kirking DM, Bartlett RH. Efficacy of patient-controlled versus conventional analgesia for postoperative pain. Clin Pharm 1985;4(1):48-52

25 Bala I, Gupta B, Bhardwaj N, Ghai B, Khosla VK. Effect of scalp block on postoperative pain relief in craniotomy patients. Anaesth Intensive Care 2006;34(2):224-227

26 Law-Koune JD, Szekely B, Fermanian C. Peuch C, Liu N, Fischler M. Scalp infiltration with bupivacaine plus epinephrine or plain ropivacaine reduces postoperative pain after supratentorial craniotomy. J Neurosurg Anesthesiol 2005;17(3):139-143

27 Biswas BK, Bithal PK. Preincision 0.25\% bupivacaine scalp infiltration and postcraniotomy pain: a randomized double-blind, placebo-controlled study. J Neurosurg Anesthesiol 2003;15(3):234-239

28 Molnár C, Simon É, Kazup Á, et al. A single preoperative dose of diclofenac reduces the intensity of acute postcraniotomy headache and decreases analgesic requirements over five postoperative days in adults: a single center, randomized, blinded trial. J Neurol Sci 2015;353(1-2):70-73

29 Williams DL, Pemberton E, Leslie K. Effect of intravenous parecoxib on post-craniotomy pain. $\mathrm{Br} \mathrm{J}$ Anaesth 2011;107(3):398-403

30 Kincaid MS, Souter MJ, Treggiari MM, Yanez ND, Moore A, Lam AM. Accuracy of transcranial Doppler ultrasonography and single-photon emission computed tomography in the diagnosis of angiographically demonstrated cerebral vasospasm. J Neurosurg 2009;110(1):67-72

31 Chowdhury T, Prabhakar H, Bithal PK, Schaller B, Dash HH. Immediate postoperative complications in transsphenoidal pituitary surgery: a prospective study. Saudi J Anaesth 2014;8(3):335-341

32 Torbic H, Forni AA, Anger KE, Degrado JR, Greenwood BC. Use of antiepileptics for seizure prophylaxis after traumatic brain injury. Am J Health Syst Pharm 2013;70(9):759-766

33 Freeman BD, Morris PE. Tracheostomy practice in adults with acute respiratory failure. Crit Care Med 2012;40(10):2890-2896

34 Stevens WR, Glazer PA, Kelley SD, Lietman TM, Bradford DS. Ophthalmic complications after spinal surgery. Spine 1997;22(12):1319-1324

35 Christie S, Thibault-Halman G, Casha S. Acute pharmacological DVT prophylaxis after spinal cord injury. J Neurotrauma 2011;28(8):1509-1514

36 Schroeder HW, Niendorf WR, Gaab MR. Complications of endoscopic third ventriculostomy. J Neurosurg 2002;96(6):1032-1040

37 Choi JU, Kim DS, Kim SH. Endoscopic surgery for obstructive hydrocephalus. Yonsei Med J 1999;40(6):600-607

38 Poston KL, Frucht SJ. Movement disorder emergencies. J Neurol 2008;255(Suppl 4):2-13 